\title{
Computing Logarithmic Capacity with Linear Programming
}

\author{
Jérémie Rostand
}

\section{CONTENTS}

1. Introduction

2. Definitions and Basic Results

3. Dirichlet Problem and Keldyš's Theorem

4. Construction of the Numerical Method

5. A Theoretical Bound on the Error

6. A Particular Case: Connected Sets

7. Examples

8. Conclusion

Acknowledgements

References
We construct an algorithm for estimating the capacity of nice compact subsets of the plane. Our method is based on a relation between capacity and Green's function together with a uniform harmonic approximation theorem. We use the simple least-square technique and, what is most important, we can obtain a bound on the error made in the estimated capacity.

\section{INTRODUCTION}

Take a compact and connected conducting body in $\mathbb{R}^{3}$, and give it a certain amount of electric charge. The charge will distribute itself so as to minimize the total electrostatic energy. The ratio between the body's charge and the value of the equilibrium potential on the surface is called the capacity.

This notion can be extended to more general sets and potentials. Here we are interested in compact subsets of $\mathbb{R}^{2}=\mathbb{C}$. In the plane it is natural to consider a repulsion law that decays with the inverse of the distance (rather than inverse squared), so the relevant potential is logarithmic. The capacity of a compact set $K$ with respect to this potential is called the logarithmic capacity. Precise definitions will be given in the next section.

Our problem is to estimate the logarithmic capacity $C(K)$ of a compact subset $K$ of the plane. It is well known that, in general, capacity is quite difficult to compute. As a matter of fact, interesting questions about logarithmic capacity are still open. One of them can be found in [Ransford 1994]. There are many inequalities involving logarithmic capacity (see [Pólya and Szegő 1951; Pommerenke and Gerd 1975], for example), but in most of them, the compact set $K$ needs to be connected. 
We shall conduct experiments using an algorithm based on a relation between Green's function and logarithmic capacity. Even though we will have to make some hypotheses on $K$, we want to keep the disconnected case in mind.

Our method transforms the problem of computing $C(K)$ into a problem of uniform harmonic approximation on $\partial K$. One of our basic tools is an approximation theorem of Keldyš. However, since the proof of this theorem is nonconstructive, it seems hard to construct an actual approximating harmonic function within a given positive $\varepsilon$. This is where it becomes experimental. We shall try to get a good approximation by employing a leastsquare technique.

One could think of using a standard finite element method to estimate the capacity of a compact set. In fact, when we know an approximation $\tilde{h}$ of the solution $h$ to the Dirichlet problem on the complement of $K$, we can compute an estimate for $C(K)$. The main problem with this method is the difficulty in obtaining a bound on the error. What we need is an estimate of $\tilde{h}-h$ at a particular point (pointwise estimate). This is fairly hard to obtain when using a finite element method (see [Wahlbin 1991, Section 10], for example). Our method is quite easy to implement and has the advantage of giving a bound on the error.

We shall proceed as follows. In Section 2 we recall the definition of logarithmic capacity and of the Green's function, some of their most familiar properties, and the interrelationship between the two concepts. Section 3 recalls the Dirichlet problem and states the approximation theorem of Keldyš needed later. In Section 4 we develop the algorithm itself. Section 5 is devoted to the derivation of error bounds for our method. In Section 6 we particularize the algorithm to connected sets, a case where many of the expressions simplify considerably. Finally, in Section 7 we look at four examples, showing how the method can be good sometimes and bad at other times. We use it to improve theoretically known bounds on $C(K)$ for some specific compact sets $K$.

\section{DEFINITIONS AND BASIC RESULTS}

If $\mu$ is a finite Borel measure with compact support in $\mathbb{C}$, its logarithmic potential is the subharmonic function

$$
p_{\mu}(z):=\int_{\mathbb{C}} \log |z-w| d \mu(w), \quad \text { for } z \in \mathbb{C} .
$$

Since the notion of potential induces a notion of energy we can define the energy of $\mu$. This is the quantity

$$
I(\mu):=\int_{\mathbb{C}} p_{\mu}(z) d \mu(z)
$$

There are several ways one could think to measure how large a subset of the complex plane is. For example, one could use diameter, area (or more generally, its measure with respect to $\mu$ ), and so on. The idea of capacity is just another way of doing that. In fact, capacity gauges how good a set is for supporting measures with high energy.

Let $K$ be a compact subset of $\mathbb{C}$. The logarithmic capacity, or simply the capacity, of $K$ is the nonnegative real number

$$
C(K):=\sup _{\nu} e^{I(\nu)}
$$

where the supremum is taken over all probability Borel measures with support in $K$. A set of capacity zero is called a polar set. If $K$ is nonpolar, the supremum is attained by a unique measure called the equilibrium measure on $K$ [Ransford 1995, Theorems 3.3.2 and 3.7.6].

Proofs of the following properties of logarithmic capacity can be found, for example, in [Ransford 1995, Theorems 5.1.2, 5.1.3, 5.1.4, 5.2.5, 5.3.4 and 5.3.5].

Theorem 2.1 (Properties of the logarithmic capacity). Let $K, K_{1}, K_{2}, \ldots$ be compact subsets of $\mathbb{C}$. Then:

(a) If $K_{1} \subset K_{2}$, then $C\left(K_{1}\right) \leq C\left(K_{2}\right)$.

(b) If $\alpha, \beta \in \mathbb{C}$, then $C(\alpha K+\beta)=|\alpha| C(K)$.

(c) $C(K)=C\left(\partial_{e} K\right)$, where $\partial_{e} K$ is the exterior boundary of $K$, that is, the boundary of the unbounded component of $\mathbb{C} \backslash K$. 
(d) If $\left(K_{n}\right)_{n \geq 1}$ is a decreasing sequence, then

$$
C\left(\bigcap_{n=1}^{\infty} K_{n}\right)=\lim _{n \rightarrow \infty} C\left(K_{n}\right) .
$$

(e) If $\left(K_{n}\right)_{n \geq 1}$ is an increasing sequence such that $K=\bigcup_{n=1}^{\infty} K_{n}$ is compact, then

$$
C(K)=\lim _{n \rightarrow \infty} C\left(K_{n}\right) .
$$

(f) If $K$ is the union of the sets $K_{n}$, and if the diameter of $K$ is less than or equal to $d$, then

$$
\frac{1}{\log (d / C(K))} \leq \sum_{n} \frac{1}{\log \left(d / C\left(K_{n}\right)\right)}
$$

(g) If $K$ has area $A$, then $C(K) \geq \sqrt{A / \pi}$.

(h) If $K$ has diameter $d$, then $C(K) \leq d / 2$.

(i) If $q$ is a polynomial of the form $q(z)=\sum_{k=0}^{n} a_{k} z^{k}$, then

$$
C\left(q^{-1}(K)\right)=\left(\frac{C(K)}{\left|a_{n}\right|}\right)^{1 / n} .
$$

Property (b) shows that capacity behaves well under translation and dilatation. Property (c) says we can fill up the holes of a set without changing its capacity. However, logarithmic capacity does not have the nice union and complement properties of a measure. This lack is certainly one of the reasons why capacity is difficult to compute.

We recall the definition of Green's function and some of its elementary properties, which we will use in Section 4.

Let $D$ be a domain of $\mathbb{C}_{\infty}$, that is, a connected open subset of the Riemann sphere. A Green's function on $D$ is a function $g_{D}: D \times D \rightarrow(-\infty, \infty]$ such that for all $w \in D$,

(a) $z \mapsto g_{D}(z, w)$ is harmonic on $D \backslash\{w\}$;

(b) $g_{D}(w, w)=\infty$, and as $z \rightarrow w$

$$
g_{D}(z, w)= \begin{cases}\log |z|+O(1) & \text { if } w=\infty \\ -\log |z-w|+O(1) & \text { if } w \neq \infty\end{cases}
$$

(c) $g_{D}(z, w) \rightarrow 0$ as $z \rightarrow \zeta$ for all $\zeta \in \partial_{\infty} D$. Here, $\partial_{\infty} D$ means the boundary of $D$ taken with respect to the sphere topology.
When a Green's function exists, $D$ is said to be a regular domain.

Proofs of the following well known facts can be found in [Ransford 1995, Section 4.4], for example.

Theorem 2.2 (Properties of the Green's function). Let $D$ be a regular domain of $\mathbb{C}_{\infty}$. Then:

(a) There exists one and only one Green's function $g_{D}$ on $D$.

(b) $g_{D}(z, w)>0$ for all $z, w \in D$.

(c) If $\left(D_{n}\right)_{n \geq 1}$ is an increasing sequence of regular domains such that $\cup D_{n}=D$, then $g_{D_{n}}(z, w) \uparrow$ $g_{D}(z, w)$ for all $z, w \in D$.

(d) $g_{D}(z, w)=g_{D}(w, z)$ for all $z, w \in D$.

There is a fundamental relation between Green's function and capacity. We shall use it in the construction of the numerical method.

Theorem 2.3. If $K$ is a compact set such that $D$, the unbounded component of $\mathbb{C}_{\infty} \backslash K$, is a regular domain, then

$g_{D}(z, \infty)=\log |z|-\log C(K)+o(1) \quad$ as $z \rightarrow \infty$.

A simple proof using Frostman's theorem can be found in [Ransford 1995, Theorem 5.2.1].

\section{DIRICHLET PROBLEM AND KELDYŠ'S THEOREM}

The Dirichlet problem has been widely studied. In this section we shall state an existence theorem which is not the most general, but which will be sufficient for our need.

Theorem 3.1. Let $D$ be a regular domain of $\mathbb{C}_{\infty}$ and let $\varphi: \partial_{\infty} D \rightarrow \mathbb{R}$ be a continuous function. Then, there exists one and only one harmonic function $h$ on $D$ such that $\lim _{z \rightarrow \zeta} h(z)=\varphi(\zeta)$ for all $\zeta \in$ $\partial_{\infty} D$.

Proof. The uniqueness of the solution follows directly from the maximum principle for harmonic functions. The existence of the solution can be proved by Perron's method. We set $h:=\sup _{u \in u} u$ where $\mathcal{U}$ is the collection of all subharmonic functions $u$ on $D$ such that $\lim \sup _{z \rightarrow \zeta} u(z) \leq \varphi(\zeta)$ for 
each $\zeta \in \partial_{\infty} D$. Then $h$ has the desired properties [Ransford 1995, Section 4.1].

In the following, we will use domains of a particular type and we would like to know if they are regular.

Theorem 3.2. Let $K$ be a compact subset of $\mathbb{C}$ such that each of its components contains at least two points. Then each component $D$ of the complement of $K$ is a regular domain.

The proof uses the concept of a barrier and we will not go into it. The interested reader can find all details in [Ransford 1995, Section 4.2].

Theorem 3.3 (Keldyš). Let $K$ be a compact subset of $\mathbb{C}$ such that $\mathbb{C} \backslash K$ has a finite number of components. Let $\Lambda$ be a subset of $\mathbb{C} \backslash K$ which has at least one point in each bounded component of $\mathbb{C} \backslash K$. Then each continuous function $\varphi: \partial K \rightarrow \mathbb{R}$ can be uniformly approximated on $\partial K$ by functions of the form $\operatorname{Re} q(z)+a \log |r(z)|$, where $a \in \mathbb{R}$ and $q$ and $r$ are rational functions such that $q, r$ and $1 / r$ have their poles in $\Lambda \cup\{\infty\}$.

The proof is based on several results including the Hahn-Banach theorem and the Riesz representation theorem. In [Ransford 1995, Theorem 6.3.3] you will find the proof of a more general statement but you can easily recover the particular case we need with the help of [Ransford 1995, Theorem 3.8.3]. Note that the Stone-Weierstrass theorem does not apply here since the collection of approximating functions does not form an algebra.

Keldyš's theorem says that it is possible to approximate a continuous function on the boundary of a compact $K$ by functions that are harmonic in a neighborhood of $K$. This is exactly what we will need in the next section.

\section{CONSTRUCTION OF THE NUMERICAL METHOD}

Under some hypotheses, it is possible to obtain a numerical approximation of the capacity of a compact set $K$. This will be done by solving a leastsquare problem.
Hypothesis 4.1. Let $K$ be a compact subset of $\mathbb{C}$ with a finite number of components $K_{j}(j=0, \ldots, n)$ such that, for each $j$, the interior $\stackrel{\circ}{K}_{j}$ is nonempty and has closure $K_{j}$.

The construction proceeds in 4 steps.

Step 1. Because of properties (b) and (c) of the logarithmic capacity from Theorem 2.1, we can suppose without loss of generality that 0 is in $\stackrel{\circ}{K}_{0}$ and that $D:=\mathbb{C}_{\infty} \backslash K$ is a domain. Define

$$
\tilde{K}:=\overline{\{z \in \mathbb{C}: 1 / z \notin K\}} .
$$

The set $\tilde{K}$ is certainly closed and, since $0 \in \stackrel{\circ}{K}$, is also bounded and therefore compact. We now pick points $\lambda_{1}, \lambda_{2}, \ldots, \lambda_{n} \in \mathbb{C}$ such that $\lambda_{j} \in \stackrel{\circ}{K}_{j}$ for $j=1, \ldots, n$. By the definition of $\tilde{K}$, the set $\Lambda:=\left\{1 / \lambda_{1}, \ldots, 1 / \lambda_{n}\right\}$ has a nonempty intersection with each bounded component of the complement of $\tilde{K}$. Let $\mathfrak{F}_{\Lambda}$ be the class of all functions of the form $\operatorname{Re} q(z)+a \log |r(z)|$, where $a \in \mathbb{R}$ and where $q$ and $r$ are rational functions such that the poles of $q, r$ and $1 / r$ are in $\Lambda$.

Keldyš's theorem (Theorem 3.3) applied with $\tilde{K}$, $\Lambda$ and $\varphi(z):=-\log |z|$ (note that $\varphi$ is continuous on $\partial \tilde{K}$ since $\operatorname{dist}(0, \partial \tilde{K})>0)$, shows that, for all $\varepsilon>0$, there exists a function $\operatorname{Re} q+a \log |r|$ in $\mathfrak{F}_{\Lambda}$ such that

$$
\|-\log |z|-\operatorname{Re} q(z)-a \log |r(z)|\|_{\partial \tilde{K}}<\varepsilon,
$$

where $\|\cdot\|_{E}$ denotes the supremum on a set $E$. We can rewrite the last inequality in the following way:

$$
\| \log |z|-\operatorname{Re} q(1 / z)-\left.a \log |r(1 / z)|\right|_{\partial K}<\varepsilon .
$$

This suggests that we define

$$
\tilde{g}(z):=\log |z|-\operatorname{Re} q(1 / z)-a \log |r(1 / z)| .
$$

As $\tilde{g}$ is continuous on the boundary of $K$, and as $D$ is a regular domain (Theorem 3.2), we deduce, with the help of Theorem 3.1, the existence of a unique function $h$, harmonic on $D$ and such that $\lim _{z \rightarrow \zeta} h(z)=\tilde{g}(\zeta)$ for all $\zeta \in \partial K$. The function $\tilde{g}-h$ has the following properties:

(a) it is harmonic on $D \backslash\{\infty\}$; 
(b) $(\tilde{g}-h)(z)=\log |z|+O(1)$ as $z \rightarrow \infty$;

(c) $(\tilde{g}-h)(z) \rightarrow 0$ as $z \rightarrow \zeta$, for all $\zeta \in \partial K$.

By the definition of the Green's function and Theorem 2.2, we deduce that $\tilde{g}-h$ is the Green's function for $D$, i.e., $g_{D}(z, \infty)=\tilde{g}(z)-h(z)$, for all $z \in D$. But we know from Theorem 2.3 that $g_{D}$ is related to the capacity of the complement of $D$ by

$$
g_{D}(z, \infty)=\log |z|-\log C(K)+o(1)
$$

as $z \rightarrow \infty$. Putting these facts together, we get

$\operatorname{Re} q(1 / z)+a \log |r(1 / z)|+h(z)=\log C(K)+o(1)$

as $z \rightarrow \infty$. The left-hand side is continuous at infinity since $h$ is harmonic at this point and

$$
\operatorname{Re} q(w)+a \log |r(w)|
$$

is continuous at 0 . Thus, we have

$$
\log C(K)=\operatorname{Re} q(0)+a \log |r(0)|+h(\infty),
$$

which is the same as

$$
C(K)=|r(0)|^{a} e^{\operatorname{Re} q(0)} e^{h(\infty)} .
$$

By the maximum principle for harmonic functions, we find $|h(\infty)| \leq\|h\|_{\partial_{\infty} D}=\|h\|_{\partial K}$. By construction, $h$ is a function that satisfies

$$
\lim _{z \rightarrow \zeta} h(z)=\tilde{g}(\zeta) \quad \text { for all } \zeta \in \partial K .
$$

Therefore, we find that $|h(\infty)| \leq\|\tilde{g}\|_{\partial K}$, and by the definition of $\tilde{g}$ this says that $|h(\infty)| \leq \varepsilon$. We have shown that

$$
C(K)=\tilde{\varepsilon}|r(0)|^{a} e^{\operatorname{Re} q(0)},
$$

where $\tilde{\varepsilon} \in\left[e^{-\varepsilon}, e^{\varepsilon}\right]$.

We have now transformed our problem of estimating the capacity of $K$ into a problem of uniform approximation of $\log |z|$ on $\partial K$ by functions of the class $\mathfrak{F}_{\Lambda}^{*}$, where $\mathfrak{F}_{\Lambda}^{*}:=\left\{z \mapsto u(1 / z): u \in \mathfrak{F}_{\Lambda}\right\}$. We know from Keldyš's theorem that this is possible, but in practice the theorem does not give a constructive way to obtain the desired approximation. In fact, it is clear that the geometry of $\partial K$ and the choice of $\lambda_{j}$ will play an important role in finding a good approximation of $\log |z|$. In the following, we shall restrict ourselves to a subspace of $\mathfrak{F}_{\Lambda}^{*}$ and a finite number of points of $\partial K$.

Step 2. If $u^{*}(z):=\operatorname{Re} q\left(\frac{1}{z}\right)+a \log \left|r\left(\frac{1}{z}\right)\right|$ is a member of $\mathfrak{F}_{\Lambda}^{*}$, then the poles and zeros of $r(z)$ are in $\Lambda=$ $\left\{1 / \lambda_{1}, \ldots, 1 / \lambda_{n}\right\}$. This implies that $r$ is of the form

$$
r(z)=c\left(z-\frac{1}{\lambda_{1}}\right)^{m_{1}} \cdots\left(z-\frac{1}{\lambda_{n}}\right)^{m_{n}},
$$

where $c$ is a constant and $m_{k}$ are integers. So,

$$
a \log |r(z)|=a \log |c|+a \sum_{k=1}^{n} m_{k} \log \left|z-\frac{1}{\lambda_{k}}\right| .
$$

Also, $q$ is a rational function whose poles are in $\Lambda$. Thus, it can be written as the sum of its principal parts at each $1 / \lambda_{k}$ plus a polynomial. If we fix two integers $N_{0}$ and $N_{1}$, the class $\mathfrak{F}_{\Lambda}^{*}\left(N_{0}, N_{1}\right)$ is defined as being the subclass of $\mathfrak{F}_{\Lambda}^{*}$ in which $q$ is of the form

$$
q(z)=\sum_{j=0}^{N_{1}} a_{j} z^{j}+\sum_{k=1}^{n} \sum_{j=1}^{N_{0}} a_{j k}\left(z-\frac{1}{\lambda_{k}}\right)^{-j},
$$

with $a_{j}, a_{j k} \in \mathbb{C}$. Therefore a typical function of $\mathfrak{F}_{\Lambda}^{*}\left(N_{0}, N_{1}\right)$ looks like

$$
\begin{aligned}
& u^{*}(z)=b_{0}+\sum_{j=1}^{N_{1}} \operatorname{Re} \frac{a_{j}}{z^{j}} \\
& +\sum_{k=1}^{n} \sum_{j=1}^{N_{0}} \operatorname{Re}\left(a_{j k}\left(\frac{z}{z-\lambda_{k}}\right)^{j}\right)+\sum_{k=1}^{n} b_{k} \log \left|\frac{z-\lambda_{k}}{z}\right|,
\end{aligned}
$$

where $a_{j}$ and $a_{j k}$ are complex constants (possibly different from the ones in the preceding equation) and $b_{k}$ are real constants. Writing $a_{j}=c_{j}+i d_{j}$ and $a_{j k}=c_{j k}+i d_{j k}$, we have the expression

$$
\begin{aligned}
& u^{*}(z)=b_{0}+\sum_{j=1}^{N_{1}} \operatorname{Re} \frac{c_{j}+i d_{j}}{z^{j}} \\
& +\sum_{k=1}^{n} \sum_{j=1}^{N_{0}} \operatorname{Re} \frac{\left(c_{j k}+i d_{j k}\right) z^{j}}{\left(z-\lambda_{k}\right)^{j}}+\sum_{k=1}^{n} b_{k} \log \left|\frac{z-\lambda_{k}}{z}\right|,
\end{aligned}
$$

where now all the constants are real. 
We shall try to approximate $\log |z|$ by functions from $\mathfrak{F}_{\Lambda}^{*}\left(N_{0}, N_{1}\right)$. In order to do so, we take an $N$ point discretization of the boundary of $K$, say $\left\{z_{l} \in\right.$ $\partial K: l=1 \ldots N\}$. As will be evident later, we must choose $N$ large enough, namely $N \geq 1+2 N_{1}+$ $n\left(2 N_{0}+1\right):=M$. We now use the least-square method to determine the "best" constants $b_{k}, c_{j}$, $d_{j}, c_{j k}$ and $d_{j k}$, i.e., those for which $u^{*}$ is as close as possible to $\log |z|$ on $\left\{z_{1}, \ldots, z_{N}\right\}$.

If $A$ is a matrix, we denote by $A_{l}^{j_{0}, j_{1}}$ the part of the $l$-th row of $A$ starting at column $j_{0}$ and ending at column $j_{1}$. We define an $N \times M$ real matrix $A$ by the following relations: for $l=1, \ldots, N$,

$$
\begin{aligned}
A_{l}^{1} & :=(1), \\
A_{l}^{2, N_{1}+1} & :=\left(\operatorname{Re} z_{l}^{-1}, \ldots, \operatorname{Re} z_{l}^{-N_{1}}\right), \\
A_{l}^{N_{1}+2,2 N_{1}+1} & :=\left(-\operatorname{Im} z_{l}^{-1}, \ldots,-\operatorname{Im} z_{l}^{-N_{1}}\right) ;
\end{aligned}
$$

for $k=1, \ldots, n$,

$$
\begin{aligned}
& A_{l}^{2 N_{1}+2+(k-1) N_{0}, 2 N_{1}+1+k N_{0}} \\
& \quad:=\left(\operatorname{Re}\left(\frac{z_{l}}{z_{l}-\lambda_{k}}\right)^{1}, \ldots, \operatorname{Re}\left(\frac{z_{l}}{z_{l}-\lambda_{k}}\right)^{N_{0}}\right), \\
& A_{l}^{2 N_{1}+2+(n+k-1) N_{0}, 2 N_{1}+1+(k+n) N_{0}} \\
& \quad:=\left(-\operatorname{Im}\left(\frac{z_{l}}{z_{l}-\lambda_{k}}\right)^{1}, \ldots,-\operatorname{Im}\left(\frac{z_{l}}{z_{l}-\lambda_{k}}\right)^{N_{0}}\right),
\end{aligned}
$$

and finally

$$
\begin{aligned}
& A_{l}^{2 N_{1}+2+2 n N_{0}, 2 N_{1}+1+n+2 n N_{0}} \\
& \quad:=\left(\log \left|\frac{z_{l}-\lambda_{1}}{z_{l}}\right|, \ldots, \log \left|\frac{z_{l}-\lambda_{n}}{z_{l}}\right|\right) .
\end{aligned}
$$

Also, let $b$ be a column vector of dimension $N$ whose $l$-th component is the real number $\log \left|z_{l}\right|$. Finally, let $x$ be an $M$-dimensional vector whose entries are

$$
\begin{aligned}
& b_{0}, \\
& c_{1}, \ldots, c_{N_{1}}, \\
& d_{1}, \ldots, d_{N_{1}}, \\
& c_{11}, \ldots, c_{N_{0} 1}, \ldots, \\
& d_{11}, \ldots, d_{N_{0} 1}, \ldots, \\
& b_{1}, \ldots, b_{n} .
\end{aligned}
$$

Step 3. Considering $x$ as a variable vector, our goal is to minimize the function $g(x):=\|A x-b\|_{2}$, where $\|\cdot\|_{2}$ is the standard Euclidean norm. We know from linear algebra that the solution-set of this problem is always nonempty. The solutions are characterized by

$$
A^{T} A x=A^{T} b .
$$

In fact, we can explicitly compute the unique solution $\tilde{x}$ with minimal norm. Set $\rho:=\operatorname{rank} A \leq M$. It is always possible to find orthogonal matrices $U_{N \times N}$ and $V_{M \times M}$ such that $U^{T} A V=\Sigma$, where $\Sigma$ is an $N \times M$ matrix defined by

$$
\Sigma_{i}^{j}:= \begin{cases}\sigma_{i} & \text { if } i=j \leq \rho, \\ 0 & \text { otherwise }\end{cases}
$$

The $\sigma_{i}$ are the positive singular values of $A$, which we can suppose to be ordered: $\sigma_{1} \geq \sigma_{2} \geq \cdots \geq$ $\sigma_{\rho}>0$.

For each vector $x$, we have

$$
\begin{aligned}
\|A x-b\|_{2}^{2} & =\left\|U^{T}\left(A\left(V V^{T}\right) x-b\right)\right\|_{2}^{2} \\
& =\left\|\Sigma V^{T} x-U^{T} b\right\|_{2}^{2} \\
& =\sum_{i=1}^{\rho}\left(\sigma_{i}\left(V^{T} x\right)_{i}-U_{i}^{T} b\right)^{2}+\sum_{i=\rho+1}^{N}\left(U_{i}^{T} b\right)^{2} .
\end{aligned}
$$

If $\tilde{x}$ is a solution to the least-square problem, it is clear that $\left(V^{T} \tilde{x}\right)_{i}=U_{i}^{T} b / \sigma_{i}$ for $i=1, \ldots, \rho$. If we set $\left(V^{T} \tilde{x}\right)_{i}=0$ for $i=\rho+1, \ldots, M$ and define $\Sigma_{M \times N}^{+}$by

$$
\left(\Sigma^{+}\right)_{i}^{j}:= \begin{cases}\sigma_{i}^{-1} & \text { if } i=j \leq \rho, \\ 0 & \text { elsewhere }\end{cases}
$$

then we get $V^{T} \tilde{x}=\Sigma^{+} U^{T} b$, which is equivalent to

$$
\tilde{x}=A^{+} b, \quad \text { with } A^{+}=V \Sigma^{+} U^{T} .
$$

We call $A^{+}$the pseudo-inverse matrix of $A$. If $\operatorname{rank} A=M$, then $A^{+}=\left(A^{T} A\right)^{-1} A^{T}$, which could also be deduced from (4.2).

Step 4. The last step is to put together everything we know so far. An approximation of $\log |z|$ on $\partial K$ can now be obtained in the following way. First, 
compute the singular values decomposition of $A$, using, for example, an iterative method [Golub and van Loan 1983]. From this, we can apply the reasoning of Step 3 to obtain a vector $\tilde{x}$ satisfying $\|A \tilde{x}-b\|_{2} \leq\|A x-b\|_{2}$ for all vectors $x$ in $\mathbb{R}^{M}$. If we recall the identification (4.1), it is clear that we have now found a function of $\mathfrak{F}_{\Lambda}^{*}\left(N_{0}, N_{1}\right)$, namely

$$
\begin{aligned}
\tilde{u}^{*}(z)= & \tilde{b}_{0}+\sum_{j=1}^{N_{1}} \operatorname{Re} \frac{\tilde{c}_{j}+i \tilde{d}_{j}}{z^{j}} \\
& +\sum_{k=1}^{n} \sum_{j=1}^{N_{0}} \operatorname{Re} \frac{\left(\tilde{c}_{j k}+i \tilde{d}_{j k}\right) z^{j}}{\left(z-\lambda_{k}\right)^{j}} \\
& +\sum_{k=1}^{n} \tilde{b}_{k} \log \left|\frac{z-\lambda_{k}}{z}\right|,
\end{aligned}
$$

which is a "good" approximation of $\log |z|$ at each $z_{l}$ of $\partial K$. If the number of points $N$ taken on the boundary of $K$ is sufficiently big, and if $\partial K$ is not too "bad", one can hope that this approximation will be good everywhere on $\partial K$ and will therefore generate a small error in the capacity.
We have already shown that

$$
C(K)=\tilde{\varepsilon} e^{\tilde{u}^{*}(\infty)},
$$

where $\tilde{\varepsilon} \in\left[e^{-\varepsilon}, e^{\varepsilon}\right]$, and where

$$
\varepsilon=\left\|\tilde{u}^{*}(z)-\log |z|\right\|_{\partial K} .
$$

Equation (4.3) shows immediately that

$$
C(K)=\tilde{\varepsilon} \exp \left(\tilde{b}_{0}+\sum_{k=1}^{n} \sum_{j=1}^{N_{0}} \tilde{c}_{j k}\right) .
$$

This concludes the construction.

\section{A THEORETICAL BOUND ON THE ERROR}

With a bit of knowledge about the geometry of $K$, one can obtain a rigorous bound for $\varepsilon$ in terms of the coefficients appearing in $\tilde{u}^{*}$. We first notice that for any holomorphic function $f(x+i y):=$ $\mu(x, y)+i \nu(x, y)$ on a domain $U$ we have

$$
\left|f^{\prime}\right|=|\nabla(\operatorname{Re} f)|=|\nabla \mu|
$$

on $U$, where $\nabla \mu$ is the gradient of $\mu$. It is now easy to obtain a crude estimate for the gradient of the two-variable real function $\varphi(x, y):=\tilde{u}^{*}(x+i y)-$ $\log |x+i y|$. With $z=x+i y$, we can write

$$
\varphi(x, y)=\operatorname{Re}\left(\tilde{b}_{0}+\sum_{j=1}^{N_{1}} \frac{\tilde{c}_{j}+i \tilde{d}_{j}}{z^{j}}+\sum_{k=1}^{n} \sum_{j=1}^{N_{0}} \frac{\left(\tilde{c}_{j k}+i \tilde{d}_{j k}\right) z^{j}}{\left(z-\lambda_{k}\right)^{j}}+\sum_{k=1}^{n} \tilde{b}_{k} \log \frac{z-\lambda_{k}}{z}-\log z\right)
$$

and using (5.1) we get

$$
\begin{aligned}
|\nabla \varphi| & =\left|\left(\tilde{b}_{0}+\sum_{j=1}^{N_{1}} \frac{\tilde{c}_{j}+i \tilde{d}_{j}}{z^{j}}+\sum_{k=1}^{n} \sum_{j=1}^{N_{0}} \frac{\left(\tilde{c}_{j k}+i \tilde{d}_{j k}\right) z^{j}}{\left(z-\lambda_{k}\right)^{j}}+\sum_{k=1}^{n} \tilde{b}_{k} \log \frac{z-\lambda_{k}}{z}-\log z\right)^{\prime}\right| \\
& =\left|-\sum_{j=1}^{N_{1}} \frac{j\left(\tilde{c}_{j}+i \tilde{d}_{j}\right)}{z^{j+1}}-\sum_{k=1}^{n} \sum_{j=1}^{N_{0}} \frac{j \lambda_{k}\left(\tilde{c}_{j k}+i \tilde{d}_{j k}\right) z^{j-1}}{\left(z-\lambda_{k}\right)^{j+1}}+\sum_{k=1}^{n} \frac{\tilde{b}_{k} \lambda_{k}}{z\left(z-\lambda_{k}\right)}-\frac{1}{z}\right| \\
& \leq \sum_{j=1}^{N_{1}} \frac{j\left|\tilde{c}_{j}+i \tilde{d}_{j}\right|}{|z|^{j+1}}+\sum_{k=1}^{n} \sum_{j=1}^{N_{0}} \frac{j\left|\lambda_{k}\right|\left|\tilde{c}_{j k}+i \tilde{d}_{j k}\right||z|^{j-1}}{\left|z-\lambda_{k}\right|^{j+1}}+\sum_{k=1}^{n} \frac{\left|\tilde{b}_{k}\right|\left|\lambda_{k}\right|}{|z|\left|z-\lambda_{k}\right|}+\frac{1}{|z|} \\
& =: \psi(z) .
\end{aligned}
$$

From this inequality, we see that the geometric properties of $K$ appearing in $\psi(z)$ are the distances between $z$ and 0 , and between $z$ and $\lambda_{k}$. 
Now suppose we have chosen $N$ points $\left\{z_{l}\right\}_{l=1}^{N}$ on the boundary of $K$, and suppose also we have a positive number $\delta$ such that

$$
\partial K \subset \bigcup_{l=1}^{n}\left\{z:\left|z-z_{l}\right|<\delta\right\} .
$$

Then, for each $z \in \partial K$, there exists an $l$ with $\left|z-z_{l}\right|<\delta$ and we have

$$
|\varphi(z)| \leq\left|\varphi\left(z_{l}\right)\right|+\delta \sup _{|\xi| \leq \delta}\left|\nabla \varphi\left(z_{l}+\xi\right)\right|
$$

Therefore,

$$
\begin{aligned}
\left\|\tilde{u}^{*}(z)-\log |z|\right\|_{\partial K} & \leq \max _{l=1, \ldots, N}\left\{\left|\varphi\left(z_{l}\right)\right|+\delta \sup _{|\xi| \leq \delta}\left|\psi\left(z_{l}+\xi\right)\right|\right\} \\
& \leq \max _{l=1, \ldots, N}\left\{\left|\tilde{u}^{*}\left(z_{l}\right)-\log \right| z_{l}||+\delta\left(\sum_{j=1}^{N_{1}} \frac{j\left|\tilde{c}_{j}+i \tilde{d}_{j}\right|}{\left(\left|z_{l}\right|-\delta\right)^{j+1}}+\sum_{k=1}^{n} \sum_{j=1}^{N_{0}} \frac{j\left|\lambda_{k}\right|\left|\tilde{c}_{j k}+i \tilde{d}_{j k}\right|\left(\left|z_{l}\right|+\delta\right)^{j-1}}{\left(\left|z_{l}-\lambda_{k}\right|-\delta\right)^{j+1}}\right.\right. \\
& \left.\left.+\sum_{k=1}^{n} \frac{\left|\tilde{b}_{k}\right|\left|\lambda_{k}\right|}{\left(\left|z_{l}\right|-\delta\right)\left(\left|z_{l}-\lambda_{k}\right|-\delta\right)}+\frac{1}{\left(\left|z_{l}\right|-\delta\right)}\right)\right\}
\end{aligned}
$$

where we are assuming that $\delta$ is small enough to satisfy $\delta<\left|z_{l}\right|$ and $\delta<\left|z_{l}-\lambda_{k}\right|$ for all $l$ and $k$.

\section{A PARTICULAR CASE: CONNECTED SETS}

If $K$ is a connected compact subset of $\mathbb{C}$, then several of the previous expressions simplify. Indeed, since $\Lambda$ is then empty, the problem reduces to approximating $\log |z|$ by functions of the type

$$
u^{*}(z)=b_{0}+\sum_{j=1}^{N_{1}} \operatorname{Re} \frac{c_{j}+i d_{j}}{z^{j}}
$$

The matrix $A$ reduces to

$$
\left(\begin{array}{ccccccc}
1 & \operatorname{Re} z_{1}^{-1} & \ldots & \operatorname{Re} z_{1}^{-N_{1}} & -\operatorname{Im} z_{1}^{-1} & \ldots & -\operatorname{Im} z_{1}^{-N_{1}} \\
\vdots & & & & & & \vdots \\
1 & \operatorname{Re} z_{N}^{-1} & \ldots & \operatorname{Re} z_{N}^{-N_{1}} & -\operatorname{Im} z_{N}^{-1} & \ldots & -\operatorname{Im} z_{N}^{-N_{1}}
\end{array}\right)
$$

and the vectors $x$ and $b$ look like

$$
\begin{aligned}
x^{T} & =\left(b_{0}, c_{1}, \ldots, c_{N_{1}}, d_{1}, \ldots, d_{N_{1}}\right), \\
b^{T} & =\left(\log \left|z_{1}\right|, \ldots, \log \left|z_{N}\right|\right) .
\end{aligned}
$$

Therefore, the capacity of $\mathrm{K}$ is given simply by

$$
C(K)=\tilde{\varepsilon} e^{\tilde{b}_{0}} .
$$

Also, with the notation of Section 5 we have

$$
\varepsilon \leq \max _{l=1, \ldots, N}\left\{\left|\tilde{u}^{*}\left(z_{l}\right)-\log \right| z_{l}||+\delta \sum_{j=1}^{N_{1}} \frac{j\left|\tilde{c}_{j}+i \tilde{d}_{j}\right|}{\left(\left|z_{l}\right|-\delta\right)^{j+1}}\right\} .
$$

\section{EXAMPLES}

We shall now look at some numerical examples of the preceding construction. We first want to get an idea about the method's performance. An easy way to do this is to use a symbolic computation software like Maple V Release 3. When this stage is completed and when we need a more efficient tool, we will use a Fortran program together with the well known Linpack library [Anderson et al. 1995] for numerical matrix computations. All calculations will be run on a Sun Sparcstation 20.

Example 7.1 (The ellipse). Consider the compact

$$
K:=\left\{z=x+i y \in \mathbb{C}: \frac{x^{2}}{9}+\frac{y^{2}}{4} \leq 1\right\} .
$$

The capacity of an ellipse with semiaxes $a$ and $b$ is known to be $\frac{1}{2}(a+b)$ [Ransford 1995, Table 5.1], 
so $C(K)=\frac{5}{2}$. This theoretical value will allow us to verify our results.

Let $e:[0,1] \rightarrow \mathbb{C}$ be the parametrization of $\partial K$ given by $e(s):=3 \cos (2 \pi s)+2 i \sin (2 \pi s)$. Fix two integers $N$ and $N_{1}$, and consider the boundary points $z_{l}=e(l / N)$ for $l=1, \ldots, N$. Now compute each entry of the matrix $A$ and the vector $b$ as described in Step 3 of the construction. Once this is done, we can solve the problem $\min _{x}\|A x-b\|_{2}$. We have two options. In general we do not know the rank of the matrix $A^{T} A$, and therefore, we do not know if it is invertible. As a consequence, we must use the singular-value decomposition method explained in the previous section. However, as $A^{T} A$ is usually invertible, we can try to solve the linear system $A^{T} A x=A^{T} b$ with a direct method. If Maple finds that $\operatorname{det} A^{T} A=0$, we will have to use the singular-value decomposition method. The advantage of solving the linear system is that it reduces the time of computation.

The listing below shows how easy it is to implement the algorithm in Maple. We define the ellipse parametrization $e$ and two procedures makeA and makeb whose function is to construct the matrix $A$ and the vector $b$ using $e, N$ and $N_{1}$. The last section has showed us that the capacity of $K$ is simply the exponential of the first component of $x$. This is what we compute in the last lines of the program.

Loading the linear algebra package and setting the precision to 16 .

$>$ with (linalg): Digits: $=16$ :

Defining the ellipse and choosing $N$ and $N 1$.

$>\mathrm{e}:=\mathrm{s}->3 * \cos (2 * \mathrm{Pi} * \mathrm{~s})+2 * \mathrm{I} * \sin (2 * \mathrm{Pi} * \mathrm{~s})$;

$>\mathrm{N}:=20: \mathrm{N} 1:=5$ :

Procedures constructing $A$ and $b$.

$>$ makeA:=proc $(A)$ local $z l, i, j$;

$>\mathrm{A}:=\operatorname{matrix}(\mathrm{N}, 1+2 * \mathrm{~N} 1)$;

$>$ for $i$ from 1 to $\mathrm{N}$ do

$>\quad z l:=\operatorname{evalf}(\mathrm{e}(\mathrm{i} / \mathrm{N}))$;

$>\quad A[i, 1]:=1$;

$>$ for $j$ from 2 to $N 1+1$ do $A[i, j]:=\operatorname{Re}(z l * *(-j+1))$; od;

$>\quad$ for $j$ from $N 1+2$ to $2 * N 1+1$ do $A[i, j]:=-\operatorname{Im}(z l * *(-j+N 1+1))$; od;

$>$ od;

$>\mathrm{A}$

$>$ end:

$>$ makeb:=proc(b) local zl,i;

$>\mathrm{b}:=\operatorname{vector}(\mathrm{N})$;

$>$ for $i$ from 1 to $N$ do $b[i]:=\operatorname{evalf}(\log (\operatorname{abs}(e(i / N))))$; od;

$>\mathrm{b}$

$>$ end:

Constructing $A$ and $b$.

$>\operatorname{makeb}(\mathrm{b})$ : $\operatorname{makeA}(\mathrm{A})$ :

Solving the linear system.

$>\mathrm{x}:=$ map (fnormal, linsolve (transpose $(\mathrm{A}) \backslash \& * \mathrm{~A}, \operatorname{transpose}(\mathrm{A}) \backslash \& * \mathrm{~b}))$ :

Computing the capacity.

$>$ cap: $=\operatorname{proc}(\mathrm{x}) \exp (\mathrm{x}[1])$ end:

$>\operatorname{cap}(\mathrm{x})$;

2.499959590588404

A Maple program to compute the capacity of an ellipse. 


\begin{tabular}{r|r|r|l|l|r} 
Pr. & $N_{1}$ & \multicolumn{1}{|c|}{$N$} & \multicolumn{1}{|c|}{ Capacity } & \multicolumn{1}{|c|}{ Error } & Time \\
\hline 16 & 5 & 20 & 2.499959590588404 & $4.0 \times 10^{-5}$ & 3.3 \\
16 & 5 & 30 & 2.500000047414742 & $4.7 \times 10^{-8}$ & 3.6 \\
24 & 5 & 50 & 2.500000000000002289858647 & $2.3 \times 10^{-14}$ & 15.2 \\
24 & 8 & 50 & 2.50000000003393085922722 & $3.4 \times 10^{-11}$ & 44.5 \\
24 & 8 & 80 & 2.49999999999999999996875 & $3.1 \times 10^{-20}$ & 98.1 \\
36 & 10 & 120 & 2.50000000000000000000000000000060743 & $6.1 \times 10^{-31}$ & 176.7
\end{tabular}

TABLE 1. Computation of the capacity of an ellipse (Example 7.1) using Maple V Release 3. The first column is the working precision (number of digits) used in all operations. The last column gives the cpu time, in seconds, needed to perform all calculations.

The result is surprising. We have chosen $N=$ 20 points on the boundary of $K$ and $N_{1}=5$ as maximal order. The error is less than $5 \times 10^{-5}$. Table 1 shows some results obtained with different choices of $N$ and $N_{1}$.

We remark two things. First, the step from row 3 to row 4 shows that we have to take a sufficient number of points on the boundary. Indeed, since we add the terms $1 / z^{6}, 1 / z^{7}$ and $1 / z^{8}$, it is possible that bigger oscillations appear in between the points of the discretization. The approximation is better at each $z_{l}$, but worse in between them. If we increase the number of points (here we go from 50 to 80 ), then the error becomes much smaller. We also note that the problem of the ellipse is rather simple. Its geometry seems to give a fairly fast convergence. This is perhaps not too surprising considering that an ellipse is a simple perturbation of a circle, for which the method gives an exact result.

Example 7.2 (The triangle). In this example the capacity is still known, but the boundary is not as nice as for the ellipse. Let $K$ be the equilateral triangle centered at 0 with one of its vertices at 1 . We have

$$
C(K)=\frac{3 \Gamma\left(\frac{1}{3}\right)^{3}}{8 \pi^{2}} \approx 0.7304992431031596 .
$$

This can be computed, for example, via conformal mappings. The Schwarz-Christoffel formula gives us an explicit expression for the conformal mapping of the complement of $K$ onto the exterior of the unit disc [Nehari 1952, Chapter IV, Section 6].
Then, we can use [Ransford 1995, Theorem 5.2.3], which links the capacity of the unit disc (which is 1) to that of $K$.

In order to estimate the capacity of $K$ using our method, it suffices to repeat each step of the first example. We define the parametrization $t:[0,1] \rightarrow$ $\mathbb{C}$ by

$t(s):= \begin{cases}1-3 s+3 s e^{\frac{2}{3} \pi i} & \text { if } 0 \leq s<\frac{1}{3} \\ (2-3 s) e^{\frac{2}{3} \pi i}+(3 s-1) e^{\frac{4}{3} \pi i} & \text { if } \frac{1}{3} \leq s<\frac{2}{3} \\ 3(1-s) e^{\frac{4}{3} \pi i}+3 s-2 & \text { if } \frac{2}{3} \leq s \leq 1\end{cases}$

We choose as before the points $z_{l}=t(l / N)$, but we have to make sure that $N$ is a multiple of 3 if we want the vertices of $K$ to be in the discretization. The rest of the algorithm is exactly the same. The results have been compiled in Table 2, where the working precision was 12 digits.

We immediately notice that the convergence is much slower in the case of a triangle. The function we want to approximate, $\log |t(s)|$, is continuous on $[0,1]$, but is not differentiable at the vertices

\begin{tabular}{r|r|l|l|r}
$N_{1}$ & \multicolumn{1}{|c|}{$N$} & Capacity & Error & Time \\
\hline 5 & 30 & 0.69288 & 0.038 & 3.8 \\
5 & 90 & 0.69107 & 0.040 & 9.7 \\
5 & 150 & 0.69093 & 0.040 & 15.6 \\
10 & 90 & 0.70166 & 0.029 & 24.1 \\
20 & 90 & 0.70761 & 0.023 & 73.2 \\
30 & 120 & 0.71156 & 0.019 & 202.3 \\
40 & 120 & 0.71808 & 0.012 & 412.8 \\
50 & 150 & 0.71563 & 0.015 & 1205.5
\end{tabular}

TABLE 2. Computation of the capacity of an equilateral triangle (Example 7.2) using Maple V Release 3 . 
of the triangle, that is, at $s=0, \frac{1}{3}, \frac{2}{3}, 1$. It is at those points that the approximation is more difficult. If we want to apply the algorithm with more points, we must use a more efficient programming language.

The Linpack Fortran library is a collection of routines that perform numerical computations with matrices in double precision. One procedure, for example, solves the least-square problem for given $A$ and $b$. It thus suffices to write a simple program in Fortran or $\mathrm{C}$ that constructs the matrix $A$ and the vector $b$ and that calls that subroutine. Table 3 shows the results obtained with this program.

One thing is now evident: the convergence is very difficult. It is impossible to go beyond $N_{1}=$ 120. Indeed, since the working precision is at most $10^{-16}$, terms like $1 / z^{130}$ would extraordinary amplify any round-off error.

What could be done to improve the rate of convergence? Here are two possibilities. First, we can change the distribution of points on $\partial K$ in order to enhance the particular situation at each vertex. Fix a number $p \in(0,1]$ and let $m_{p}:[0,1] \rightarrow[0,1]$

\begin{tabular}{r|r|r|r|r}
$N_{1}$ & \multicolumn{1}{|c|}{$N$} & Capacity & Error & Time \\
\hline 50 & 150 & 0.71563 & 0.015 & 0.3 \\
50 & 210 & 0.71428 & 0.015 & 0.4 \\
50 & 390 & 0.71424 & 0.015 & 0.9 \\
50 & 600 & 0.71423 & 0.015 & 1.5 \\
60 & 600 & 0.71552 & 0.015 & 1.9 \\
70 & 600 & 0.71628 & 0.015 & 2.5 \\
80 & 600 & 0.71702 & 0.013 & 3.2 \\
80 & 900 & 0.71688 & 0.014 & 4.9 \\
90 & 900 & 0.71707 & 0.013 & 6.3 \\
100 & 900 & 0.71718 & 0.013 & 7.4 \\
100 & 1200 & 0.71799 & 0.013 & 11.9 \\
100 & 1500 & 0.71715 & 0.013 & 16.3 \\
100 & 1800 & 0.71737 & 0.013 & 21.6 \\
100 & 2100 & 0.71758 & 0.013 & 26.1 \\
110 & 2100 & 0.71767 & 0.013 & 31.2 \\
120 & 2100 & 0.71764 & 0.013 & 36.3
\end{tabular}

TABLE 3. Computation of the capacity of an equilateral triangle using Linpack. be the homeomorphism defined by the condition that the derivative $m_{p}^{\prime}(x)$ equals

$$
\begin{cases}p & \text { if } x \in\left(0, \frac{1}{9}\right) \cup\left(\frac{2}{9}, \frac{4}{9}\right) \cup\left(\frac{5}{9}, \frac{7}{9}\right) \cup\left(\frac{8}{9}, 1\right), \\ 3-2 p & \text { if } x \in\left(\frac{1}{9}, \frac{2}{9}\right) \cup\left(\frac{4}{9}, \frac{5}{9}\right) \cup\left(\frac{7}{9}, \frac{8}{9}\right)\end{cases}
$$

The new parametrization $t \circ m_{p}$ is "slower" near of the triangle vertices and hence, more points will be chosen in the neighborhood of each vertex.

A second idea to increase the performance of this method is to enlarge the space of functions onto which we project $\log |z|$. In the construction of the algorithm we used the minimal set $\Lambda$, that is, a set with exactly one point in the interior of each component of $K$ that does not contain 0 . However, nothing prevents us from taking more points in $\Lambda$, and therefore obtaining a wider set of functions with which to approximate $\log |z|$.

If we combine these two ideas, we get some somewhat better results. For example, take $\lambda_{1}=1-d$, $\lambda_{2}=e^{2 \pi i / 3} \lambda_{1}$ and $\lambda_{3}=e^{2 \pi i / 3} \lambda_{2}$ for a certain $d \in$ $(0,1)$. Now try the algorithm for different values of $d$ and $p$. The results are shown in Table 4 .

The last example shows that even in a simple case like a triangle, the numerical computations can be difficult. We shall now look at examples where the capacity is not explicitly known. We shall try to estimate the error and to improve the standard estimates.

Example 7.3. Let $K$ be the compact set shown in Figure 1, which is the union of the unit circle centered at the origin, the unit half-circle $(\operatorname{Im} z \geq 0)$ centered at $3+0 i$, and the unit half-circle $(\operatorname{Re} z \geq$ 0) centered at $3 i$.

The capacity of $K$ can be estimated with inequalities from the theory. Indeed, the diameter of $K$ is $4 \sqrt{2}$, so property (h) of Theorem 2.1 shows that $C(K) \leq 2 \sqrt{2} \leq 2.82842713$. On the other hand, property $(\mathrm{g})$ of the same theorem leads to $C(K) \geq \sqrt{2} \geq 1.41421356$. 


\begin{tabular}{r|c|c|c|c|c|c|r}
$N_{0}$ & $N_{1}$ & $N$ & $d$ & $p$ & Capacity & Error & Time \\
\hline 0 & 50 & 600 & - & 0.80 & 0.71493 & $1.6 \times 10^{-2}$ & 1.4 \\
0 & 50 & 600 & - & 0.60 & 0.71733 & $1.3 \times 10^{-2}$ & 1.4 \\
0 & 50 & 600 & - & 0.50 & 0.71745 & $1.3 \times 10^{-2}$ & 1.4 \\
0 & 50 & 600 & - & 0.40 & 0.72501 & $5.5 \times 10^{-3}$ & 1.4 \\
0 & 50 & 600 & - & 0.35 & 0.73076 & $2.6 \times 10^{-4}$ & 1.4 \\
0 & 50 & 600 & - & 0.30 & 0.73683 & $6.3 \times 10^{-3}$ & 1.4 \\
5 & 50 & 600 & 0.70 & 0.35 & 0.73478 & $4.3 \times 10^{-3}$ & 2.3 \\
5 & 50 & 600 & 0.50 & 0.35 & 0.72723 & $3.3 \times 10^{-3}$ & 2.3 \\
5 & 50 & 600 & 0.30 & 0.35 & 0.72689 & $3.6 \times 10^{-3}$ & 2.3 \\
5 & 50 & 600 & 0.10 & 0.35 & 0.72845 & $2.0 \times 10^{-3}$ & 2.3 \\
5 & 50 & 600 & 0.05 & 0.35 & 0.72983 & $6.7 \times 10^{-4}$ & 2.3 \\
5 & 50 & 600 & 0.03 & 0.35 & 0.73066 & $1.6 \times 10^{-4}$ & 2.3 \\
8 & 50 & 600 & 0.03 & 0.35 & 0.73065 & $1.5 \times 10^{-4}$ & 2.9 \\
10 & 50 & 600 & 0.03 & 0.35 & 0.73062 & $1.2 \times 10^{-4}$ & 3.3 \\
12 & 60 & 900 & 0.03 & 0.35 & 0.73042 & $7.9 \times 10^{-5}$ & 7.5
\end{tabular}

TABLE 4. Computation of the capacity of an equilateral triangle using Linpack, with a nonuniform parametrization and a variable $\Lambda$ set.

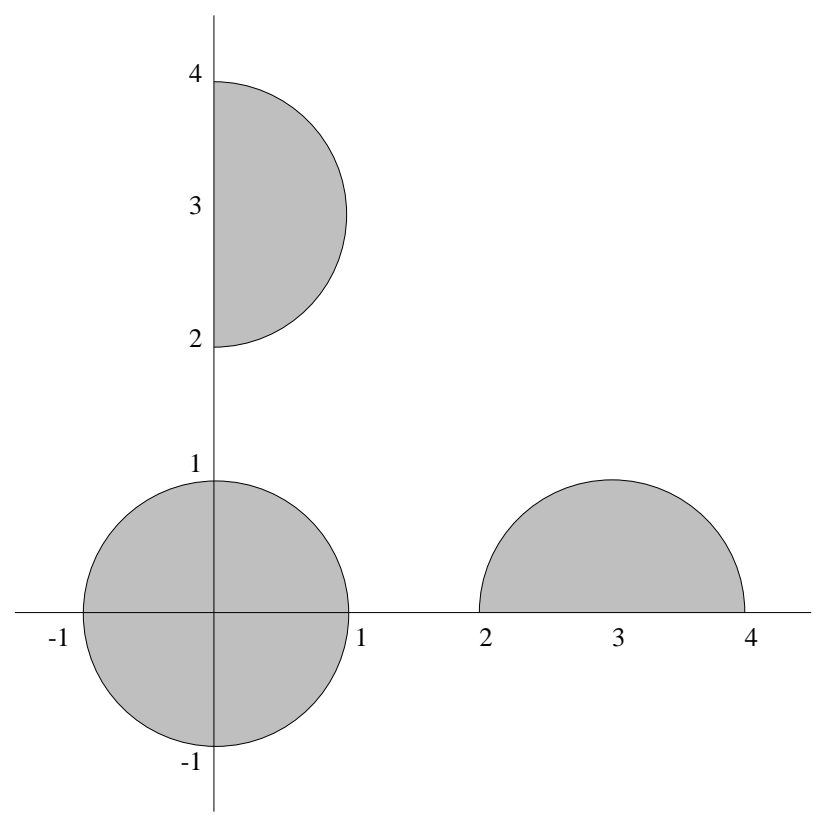

FIGURE 1. The compact $K$ of Example 7.3.

The boundary of $K$ is made of 3 disjoint curves. Let $d:[0,3] \rightarrow \mathbb{C}$ be the function

$$
d(x):= \begin{cases}e^{2 \pi i x} & \text { if } 0 \leq x \leq 1 \\ 4 x-2 & \text { if } 1<x \leq \frac{3}{2} \\ 3+e^{2 \pi i(x-3 / 2)} & \text { if } \frac{3}{2}<x \leq 2 \\ 3 i+e^{2 \pi i(x-9 / 4)} & \text { if } 2<x \leq \frac{5}{2} \\ 14 i-4 i x & \text { if } \frac{5}{2}<x \leq 3\end{cases}
$$

As $x$ runs from 0 to 3 , the parametrization $d$ describes the boundary of $K$, with discontinuities at 1 and 2 .

If $N$ is a multiple of 3 , we choose the points $z_{l}=$ $d(3 l / N)$ for $l=1, \ldots, N$. Let $n$ be the number of points in $\Lambda$ (we must have $n \geq 2$ in order to be sure that $\mathfrak{F}_{\Lambda}^{*}$ is dense in the set of continuous functions on $\partial K)$. Let also $\lambda_{1}=3+\frac{1}{2} i$ and $\lambda_{2}=\frac{1}{2}+3 i$ be two points in $\stackrel{\circ}{K}$. For the purpose of getting a first idea of what is going on in this example, we choose the parameters, rather arbitrarily, as $n=2$, $N=600, N_{0}=5$, and $N_{1}=20$. The result is $C(K)=2.17844$, computed in $0.6 \mathrm{~s}$.

Since we do not know the exact value of $C(K)$, we may look at the error graph, that is, the graph of $u^{*}(d(x))-\log |d(x)|$ on the interval $[0,3]$. With more than 12000 points in $[0,3]$ we get Figure 2 . This immediately gives us a bound on the maximum possible error. Indeed, we see that

$$
\left\|u^{*}(z)-\log |z|\right\|_{\partial K} \leq 0.15
$$

and so $C(K)$ is in the interval

$$
\left[e^{-0.15} 2.17844, e^{0.15} 2.17844\right]=[1.875,2.531] .
$$

This is a better estimate than the theoretical one stated above. 


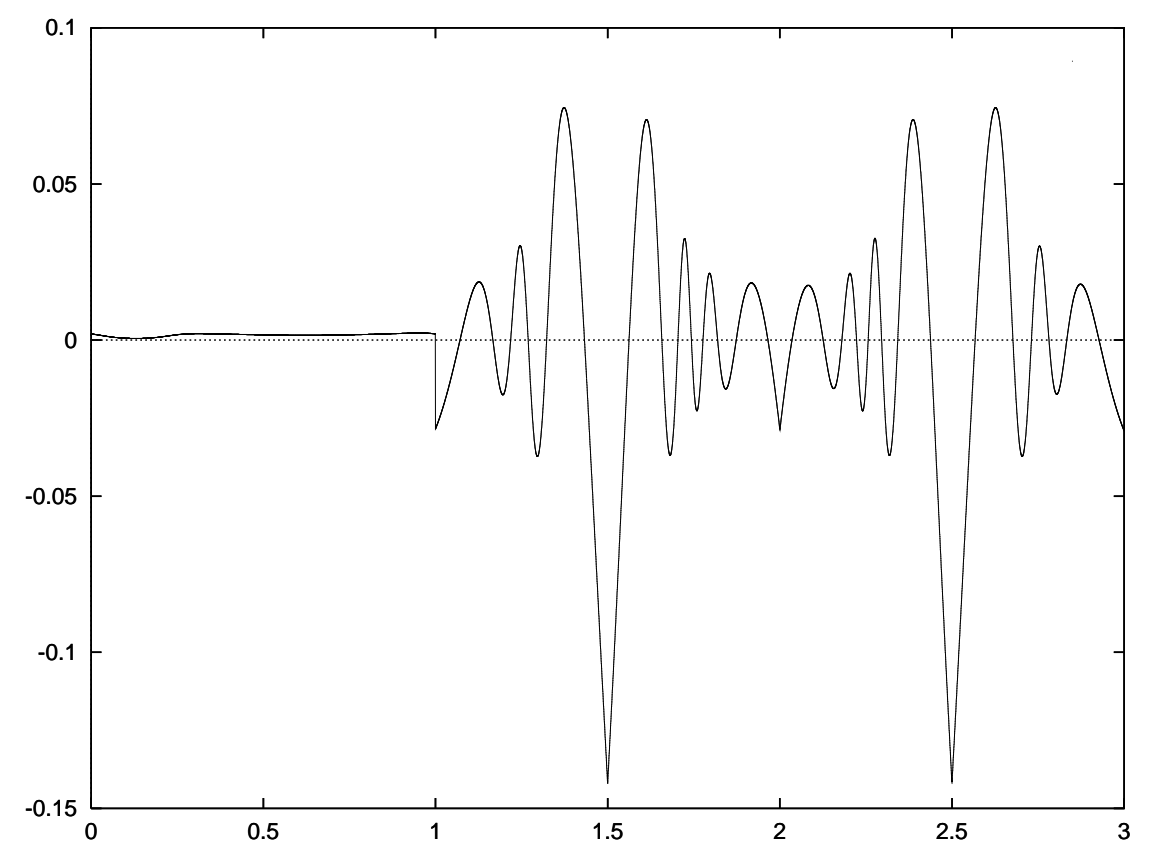

FIGURE 2. Error graph for the compact of Example 7.3, with $n=2, N=600, N_{0}=5, N_{1}=20$.

We could also get a rigorous bound on the error by using the calculus made in Section 5 . As the $N=600$ points are uniformly distributed on $\partial K$ (in the sense of the parametrization $d$ ), we can take $\delta \geq \sin (3 \pi / N)=\sin (\pi / 200) \geq 0.015707$. With this choice of $\delta$ we have

$$
\left\|u^{*}(z)-\log |z|\right\|_{\partial K} \leq 0.151874
$$

which is pretty close to the previous bound.

Let's look back at the error graph. We notice that the error on the interval $[0,1]$ is quite small (of order $1 \times 10^{-3}$ ), and hence, the approximation of $\log |z|$ on the unit circle is easy - no surprise here! However, the points $x=1.5$ and $x=2.5$ are comparatively bad. They correspond to the junction of the circle arcs with the segments where the distance from the origin is the largest $(z=4$ and $z=4 i)$. At those points, $\log |z|$ is more difficult to approximate.

To solve this problem, we repeat the idea of the previous example. We first reparametrize the boundary to get more points near the corners of $K$. We compose $d$ with the homeomorphism $m_{p}$ :
$[0,3] \rightarrow[0,3]$ determined by the condition that $m_{p}^{\prime}(x)$ equals

$$
\begin{cases}1 & \text { if } x \in(0,1), \\ p & \text { if } x \in\left(1, \frac{7}{6}\right) \cup\left(\frac{4}{3}, \frac{5}{3}\right) \cup\left(\frac{11}{6}, \frac{13}{6}\right) \cup\left(\frac{7}{3}, \frac{8}{3}\right) \\ 3-2 p & \text { otherwise. }\end{cases}
$$

We also choose some extra $\lambda_{k}$. We try several values of the parameters and try to minimize the error by looking at the graph error. We are able to reduce by a factor of 10 the error on $C(K)$. Indeed, if we define $\lambda_{3}=3.95+0.05 i, \lambda_{4}=0.05+3.95 i$, $\lambda_{5}=2.05+0.05 i$ and $\lambda_{6}=0.05+2.05 i$, we find that the choices $n=6, N=4800, N_{0}=20, N_{1}=10$, and $p=0.1$ give $C(K)=2.196961$, in $130.9 \mathrm{~s}$.

Figure 3 shows a plot of the function

$$
u^{*}\left(d\left(m_{p}(x)\right)\right)-\log \left|d\left(m_{p}(x)\right)\right|
$$

on $[0,3]$. The curve is bounded above by $5 \times 10^{-3}$ and bounded below by $-1.05 \times 10^{-2}$. Hence, if we add $2.75 \times 10^{-3}$ to the function $u^{*}$, the maximum error reduces to $7.75 \times 10^{-3}$, and $C(K)$ lies in 


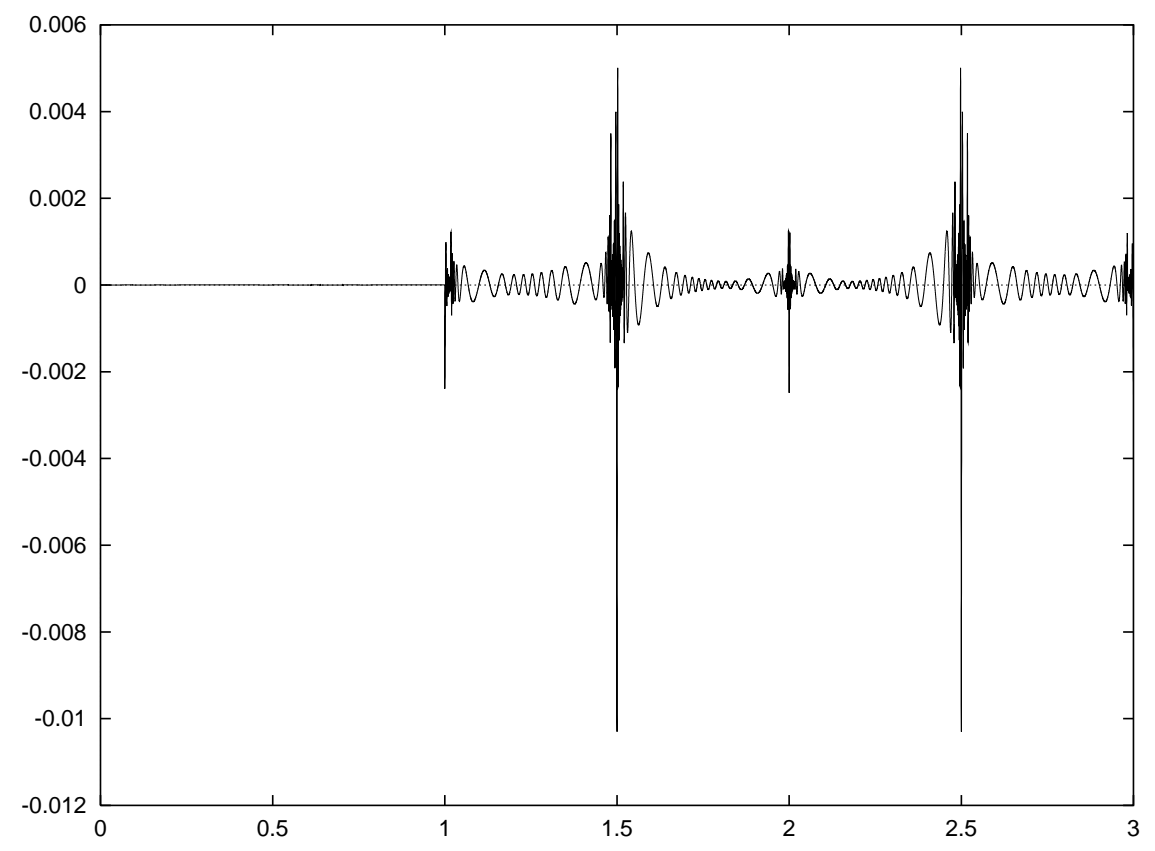

FIGURE 3. Error graph for the compact of Example 7.3, with $n=6, N=4800, N_{0}=20, N_{1}=10, p=0.1$.

$$
\begin{aligned}
& {\left[e^{-0.0075} 2.196961 e^{0.00275}, e^{0.0075} 2.196961 e^{0.00275}\right] } \\
&= {[2.18655,2.21960] . }
\end{aligned}
$$

Now let's try to get a rigorous bound as before. Taking $\delta \geq \sin 3 \pi(3-2 p) / N \geq 0.005497759$ we get the estimate

$$
\left|u^{*}\left(d\left(m_{p}(x)\right)\right)-\log \right| d\left(m_{p}(x)\right)|| \leq 4467.75 .
$$

It is easy to understand why we obtain such a big number. The problem comes from the choices of the extra $\lambda_{k}$ and from the degree of the principal parts at each $\lambda_{k}$, that is $N_{0}$. Indeed, there are points of the discretization for which $\left|z_{l}-\lambda_{k}\right| \approx$ 0.05 for $k \geq 3$ and then the terms

$$
\frac{j\left|\lambda_{k}\right|\left|\tilde{c}_{j k}+i \tilde{d}_{j k}\right|\left(\left|z_{l}\right|+\delta\right)^{j-1}}{\left(\left|z_{l}-\lambda_{k}\right|-\delta\right)^{j+1}}
$$

in the estimate of $\varepsilon$ can become very large, especially when $j$ approaches $N_{0}$. We need to decrease $N_{0}$. A good rigorous bound can be obtain with the choices $n=6, N=9999, N_{0}=5, N_{1}=5$, and $p=1$; we get $C(K)=2.196961$ with $\varepsilon<0.0229$.
Example 7.4 (Discs). As a last example, take the compact

$$
\begin{aligned}
K & =\{4\} \cup \bigcup_{k=0}^{\infty} \bar{\Delta}\left(4-\frac{1}{2^{k-2}}, \frac{1}{2^{k}}\right) \\
& =\{4\} \cup \bar{\Delta}(0,1) \cup \bar{\Delta}\left(2, \frac{1}{2}\right) \cup \bar{\Delta}\left(3, \frac{1}{4}\right) \cup \cdots,
\end{aligned}
$$

where $\bar{\Delta}(z, r)$ is the closed disk of radius $r$ centered at $z$. (See Figure 4.) Our goal is to improve the bounds on $C(K)$ that we know from the theory.

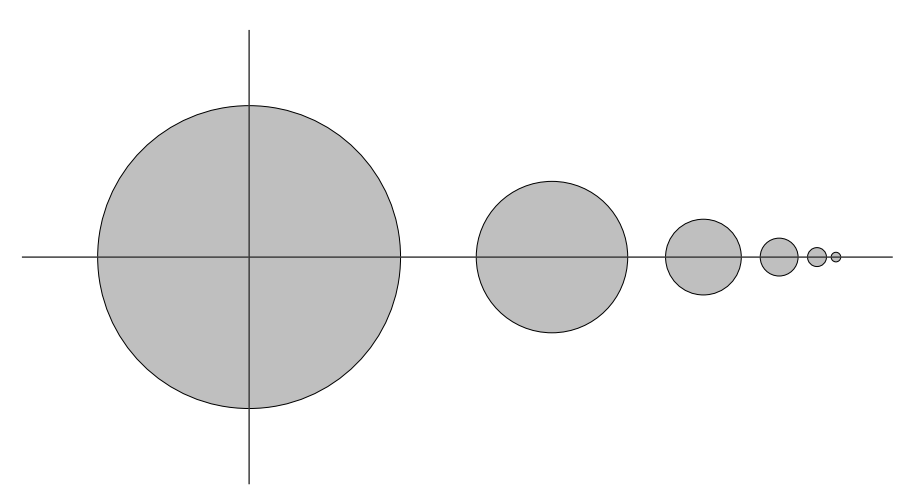

FIGURE 4. The compact $K$ of Example 7.4. 


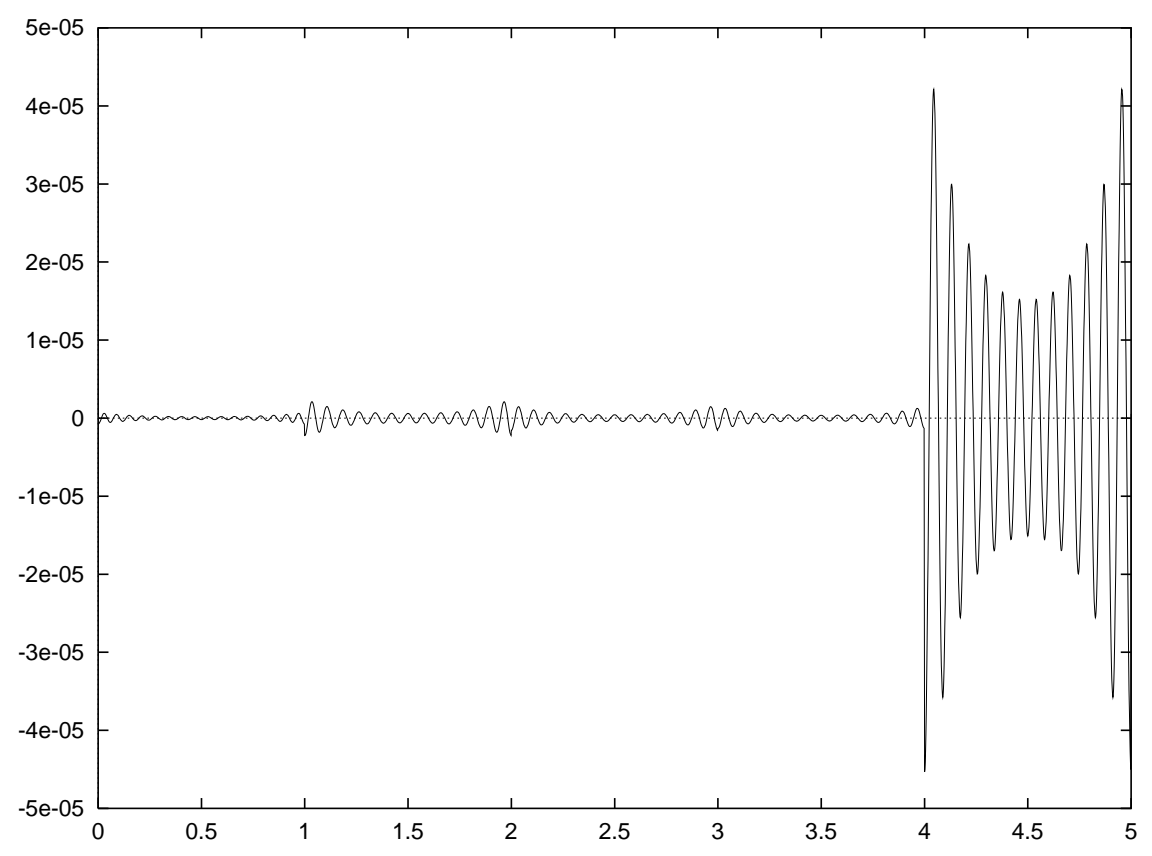

FIGURE 5. Error graph for the compact of Example 7.4, with $n=5, N=1000, N_{0}=12, N_{1}=15$.

Part $(\mathrm{g})$ of Theorem 2.1 readily shows that

$$
C(K) \geq \sqrt{\frac{1}{\pi} \sum_{k=0}^{\infty} \pi \frac{1}{2^{2 k}}}=\frac{2}{\sqrt{3}} \approx 1.154701 .
$$

Getting an upper bound for $C(K)$ is more difficult. Let $K_{n}$ be the union of the first $n$ discs (so that $\left.K_{1}:=\bar{\Delta}(0,1)\right)$. The first idea is to use part (f) with $K_{n}$. Unfortunately, the series diverges and this inequality gives nothing better than the fact that $C(K) \leq \frac{5}{2}$, which can be deduced from property (h). However, if we use part (b) with $\alpha=\frac{1}{2}$ and part (f) with the two compact sets $K_{1}$ and $K \backslash K_{1}$, we find a better estimate, namely

$$
C(K) \leq 5 \times 4^{-(\log 5) /(\log 20)} \approx 2.374199 .
$$

We now want to improve these bounds.

If we know the capacity of $K_{n}$, we can repeat the last argument with $K_{n}$ and $K \backslash K_{n}$ to get a better upper bound. Each $K_{n}$ is an admissible compact for the algorithm of the previous section. Let us take $\lambda_{k}=4-2^{-(k-2)}$. A first computation with $n=5, N=1000, N_{0}=12$, and $N_{1}=15$ gives
$C\left(K_{5}\right)=1.581659$ in $3.9 \mathrm{~s}$. Figure 5 shows that the maximum possible error is $5 \times 10^{-5}$.

Before increasing the number of discs, we have to wonder how far this will make sense. With the given working precision, the computer is incapable of distinguishing between any of the discs after the fiftieth. Thus, we have to take $n \leq 50$. Also, it is probable that even low order terms will be gigantic as $n$ increases. In order to avoid this problem, we generalize the algorithm by letting $N_{0}$ vary with $\lambda_{k}$. For example, if $n$ is small, we can take several terms (just as in the previous computation), but as $n$ increases, we must decrease $N_{0}$. We take $N=$ 1200 and $N_{1}=12$, and we apply this new idea.

The results are displayed in Table 5. In each computation, the order $N_{0}$ of the principal part around $\lambda_{k}$ varies from 12 to 2 as $\lambda_{k}$ gets closer to 4 . The column labeled Error is the maximum value seen on the graph of $\left|u^{*}(z)-\log \right| z||$ where $z \in \partial K_{n}$. For example, Figure 6 display the error graph for $n=46$. More than 100000 points have been calculated to draw this curve which is continuous on each interval $(n-1, n)$, the parametrization domain of disc number $n$. Hence, by the 


\begin{tabular}{r|c|c|r||c|c|c|c}
\multicolumn{1}{r|}{$n$} & $C\left(K_{n}\right)$ & Error & Time & $n$ & $C\left(K_{n}\right)$ & Error & Time \\
\hline 8 & 1.6084424559 & $3 \times 10^{-4}$ & 9.6 & 28 & 1.6116896006 & $8 \times 10^{-6}$ & 42.1 \\
10 & 1.6109518683 & $2 \times 10^{-4}$ & 12.8 & 30 & 1.6116895940 & $1 \times 10^{-5}$ & 44.8 \\
12 & 1.6115219754 & $6 \times 10^{-5}$ & 16.4 & 32 & 1.6116896039 & $6 \times 10^{-6}$ & 47.2 \\
14 & 1.6116515135 & $6 \times 10^{-5}$ & 19.8 & 34 & 1.6116896099 & $5 \times 10^{-6}$ & 50.3 \\
16 & 1.6116809506 & $3 \times 10^{-5}$ & 23.5 & 36 & 1.6116896069 & $5 \times 10^{-6}$ & 52.4 \\
18 & 1.6116876417 & $3 \times 10^{-5}$ & 27.0 & 38 & 1.6116895999 & $8 \times 10^{-6}$ & 54.5 \\
20 & 1.6116891577 & $3 \times 10^{-5}$ & 30.3 & 40 & 1.6116895603 & $2 \times 10^{-5}$ & 56.9 \\
22 & 1.6116895080 & $8 \times 10^{-6}$ & 33.6 & 42 & 1.6116895936 & $2 \times 10^{-5}$ & 59.5 \\
24 & 1.6116895789 & $8 \times 10^{-6}$ & 36.5 & 44 & 1.6116896052 & $5 \times 10^{-6}$ & 60.5 \\
26 & 1.6116896064 & $5 \times 10^{-6}$ & 39.6 & 46 & 1.6116896120 & $4 \times 10^{-6}$ & 62.5
\end{tabular}

TABLE 5. Computation of the capacity of the set of Example 7.4 using the adaptive method.

reasoning already described, we have

$$
1.611685 \leq C(K) \leq 1.674152 .
$$

Thus the theoretical upper bound computed before was rather imprecise. Since we are using the same technique here, we can guess that in fact $C(K)$ is much closer to $C\left(K_{n}\right)$ than suggested by the maximal error.

We wonder now if we could get a rigorous bound on the error using the technique of Section 5. Before starting any computation, we mention some facts. First, suppose we have $M$ equidistributed points on a circle of radius $r$. Then, if we want to cover this circle with discs of radius $\delta$ centered at each of the $M$ points, we need to choose $\delta \geq$ $2 r \sin (\pi /(2 M))$. Since $r=2^{1-k}$ for disc number $k$, we see that $\delta$ varies a lot from a part of $K$ to another. This suggests that we use several $\delta$, for example one for each disc:

$$
\delta_{k}=2^{2-k} \sin \frac{\pi}{2 N / n}, \quad \text { for } k=1 \ldots n
$$

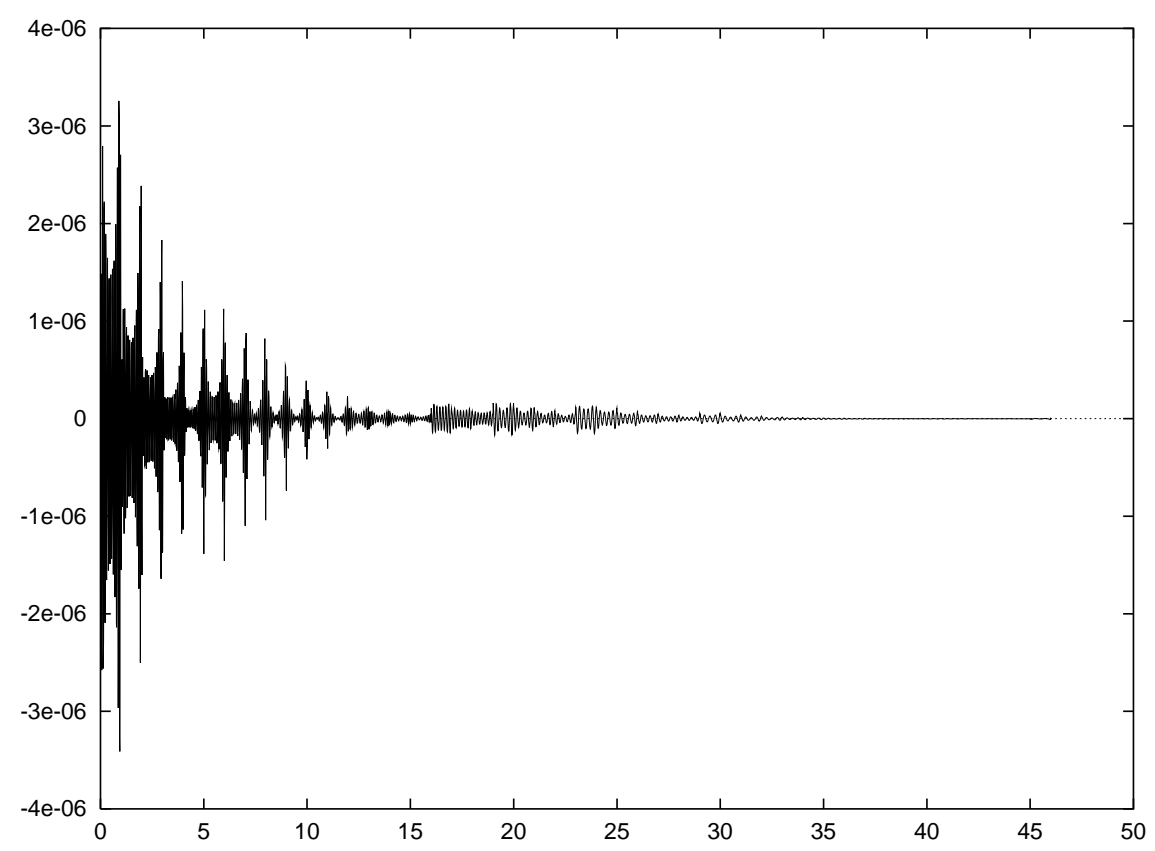

FIGURE 6. Error graph for the compact of Example 7.4, with $n=46, N=1000, N_{1}=12, N_{0}$ variable. 
However, even with this improvement, there is still a difficulty. In the expression of the upper bound for $\varepsilon$, we have terms like

$$
\frac{j\left|\lambda_{k}\right|\left|\tilde{c}_{j k}+i \tilde{d}_{j k}\right|\left(\left|z_{l}\right|+\delta\right)^{j-1}}{\left(\left|z_{l}-\lambda_{k}\right|-\delta\right)^{j+1}} .
$$

These terms are very large when $k=n-1$. Indeed, we then have $\left|z_{l}-\lambda_{k}\right|=2^{-n}$ for each point on the last circle and since $j$ is running from 1 to $N_{0}$ we have factors of order

$$
\left(2^{-n}-\delta\right)^{-N_{0}-1}
$$

With our previous choice of $\delta$, we get

$$
\begin{aligned}
\left(2^{-n}-\delta\right)^{-N_{0}-1} & =2^{n\left(N_{0}+1\right)}\left(1-4 \sin \frac{n \pi}{2 N}\right)^{-N_{0}-1} \\
& \approx 2^{n\left(N_{0}+1\right)}\left(1+\frac{2\left(N_{0}+1\right) n \pi}{N}\right) .
\end{aligned}
$$

Therefore, we cannot expect a good bound on the error when $n$ and $N_{0}$ are big. We can try to compensate with a high number of points, but since the linear system is huge (due to the number of $\lambda_{k}$ ) it now becomes impracticable. In fact, with the hardware we are using it is difficult to exceed 5000 points.

Here is an example of the rigorous bound obtained with $n=10, N=4800, N_{0}=5$ and $N_{1}=5$ :

$$
C\left(K_{10}\right)=1.6109514835, \quad \varepsilon \leq 6.7 \times 10^{-3} .
$$

\section{CONCLUSION}

The method developed in this paper was based on an approximation theorem from Keldyš. This theorem asserts that $\log |z|$ can be uniformly approximated on the compact set considered above by harmonic functions of the class $\mathfrak{F}_{\Lambda}$. Unfortunately, the proof of the theorem is nonconstructive, and given a positive $\varepsilon$, we do not have any algorithm to construct a harmonic function $\varepsilon$-close to $\log |z|$. The idea here was to project the function $\log |z|$ on a subspace $\mathfrak{F}_{\Lambda}^{*}\left(N_{0}, N_{1}\right)$, taking a finite number of points on $\partial K$. Our projection was the least-square method. We do not really know if it is always possible to approximate $\log |z|$ in this way. That was a hypothesis of the algorithm. This suggests a direction for further research. Indeed, it would be interesting to know if the subspaces $\mathfrak{F}_{\Lambda}^{*}\left(N_{0}, N_{1}\right)$ really allow approximation of $\log |z|$. How should $N_{0}$ and $N_{1}$ increase and what is the best way of picking the boundary points? Also, one can ask if it is possible to improve the argument of Section 5 in order to obtain a better rigorous bound on the maximum of $\left|u^{*}(z)-\log \right| z||$.

The reader may have noticed that if we knew an explicit harmonic function $h$ equal to $\log |z|$ on $\partial K$ (Dirichlet problem), then the problem of finding $C(K)$ would be solved. As mentioned before, one might think of using a standard algorithm like the finite element method to obtain an approximation of $h$. However, since the approximation we obtain is not itself harmonic we cannot apply the maximum principle and it is much more difficult to obtain a bound on the error we make and hence on the error on $C(K)$.

\section{ACKNOWLEDGEMENTS}

This work formed part of a masters' thesis at Université Laval, and I would like to thank my supervisor Professor T. J. Ransford for the helpful discussions we had together.

\section{REFERENCES}

[Anderson et al. 1995] E. Anderson et al., LAPACK user's guide, 2nd ed., Philadelphia: Society for Industrial and Applied Mathematics, 1995. See http:// www.netlib.org/lapack/lug/lapack_lug.html.

[Golub and van Loan 1983] G. H. Golub and C. F. van Loan, Matrix computations, The Johns Hopkins University Press, Baltimore, Maryland 21218, 1983.

[Nehari 1952] Z. Nehari, Conformal mapping, McGrawHill, Toronto, 1952.

[Pólya and Szegő 1951] G. Pólya and G. Szegö, Isoperimetric inequalities in mathematical physics, Annals of Mathematics Studies 27, Princeton Univ. Press, Princeton, 1951. 
[Pommerenke and Gerd 1975] C. Pommerenke and J. Gerd, Univalent functions, Studia mathematica 25, Vandenhoeck und Ruprecht, Göttingen, 1975.

[Ransford 1994] T. J. Ransford, "A lower bound for capacity", pp. 136-138 in Linear and complex analysis problem: Problem book 3, part II, edited by V. P. Havin and N. K. Nikolski, Lecture Notes in Math. 1574, Springer, New York, 1994.
[Ransford 1995] T. J. Ransford, Potential theory in the complex plane, Cambridge Univ. Press, Cambridge, 1995.

[Wahlbin 1991] L. B. Wahlbin, "Local behavior in finite element methods", pp. 353-522 in Handbook of numerical analysis II: Finite Element Methods, part 1, edited by P. G. Ciarlet and J. L. Lions, Elsevier, North-Holland, 1991.

Jérémie Rostand, Département de mathématiques et statistique, Université Laval, Québec, PQ, G1K 7P4, Canada (jrostand@mat.ulaval.ca)

Received August 13, 1996; accepted in revised form March 13, 1997 